\title{
Avaliação clínica da ablação uveal intravítrea com gentamicina em cães portadores de glaucoma crônico
}

[Clinical evaluation of intravitreal uveal ablation with gentamicin in chronic glaucomatous dogs]

\author{
J.L.V. Chiurciu ${ }^{1}$, C.V.S. Brandão ${ }^{1}$, J.J.T. Ranzani ${ }^{2}$, D.N. Cremonini ${ }^{1}$, J.A. Crocci $^{3}$ \\ ${ }^{1}$ Aluno de pós-graduação - UNESP - Botucatu, SP \\ ${ }^{2}$ Faculdade de Medicina Veterinária e Zootecnia - UNESP \\ Distrito de Rubião Júnior, $\mathrm{s} / \mathrm{n}$ \\ 18618-000 -Botucatu, SP \\ ${ }^{3}$ Instituto de Biocências - UNESP - Botucatu, SP
}

\begin{abstract}
RESUMO
Investigou-se, clinicamente o resultado da ablação uveal intravítrea em 13 olhos cegos de cães com glaucoma crônico unilateral. Os olhos acometidos foram submetidos à ablação uveal intravítrea, por meio de injeção na câmara vítrea de $0,5 \mathrm{ml}$ de sulfato de gentamicina $(40 \mathrm{mg} / \mathrm{ml})$ associado a $0,3 \mathrm{ml}$ de fosfato de dexametasona $(4 \mathrm{mg} / \mathrm{ml})$. As variáveis clinicas oftálmicas foram quali-quantificadas em escores, por até 48 semanas do pós-operatório; além de aspectos relacionados à dor, como variações do apetite e peso corporal. Nos sinais clínicos, de secreção ocular, blefaroespasmo, quemose, hifema e pigmentação, neovascularização, pannus e variações de apetite e peso corporal, não se notaram diferenças significativas entre os momentos. A ablação uveal intravítrea diminuiu a hiperemia conjuntival, porém acarretou aumento de opacidade corneana. A associação da ablação com antiinflamatórios tópico e sistêmico indicou não se tratar de procedimento doloroso.
\end{abstract}

Palavras-chave: cão, ablação farmacológica, gentamicina, glaucoma

\begin{abstract}
The purpose of the study was to investigate the clinical alterations of intravitreal uveal ablation. Thirteen irreversible blind canine eyes, presenting unilateral chronic glaucoma. All the glaucomatous eyes were submitted to intravitreal uveal ablation but the injection of $0.5 \mathrm{ml}$ of gentamicin sulfate solution $(40 \mathrm{mg} / \mathrm{ml})$ associated with $0.3 \mathrm{ml}$ of dexametasone phosphate $(4 \mathrm{mg} / \mathrm{ml})$ through the vitreous chamber. The oftalmic clinical variables were evaluated and classified in scores until 48 weeks after surgery. Clinical signs of pain, like apetite variations and body weight were also evaluated. Clinical signs of ocular discharge, blepharoespasm, quemosis, hifema and pigmentation, neovascularization, pannus and appetite variations and body weight did not show significant differences. The intravitreal uveal ablation reduced the conjunctival hyperemia, however caused increase in corneal opacity. The association of ablation with topic and sistemic antiflamatories was not a painful procedure.
\end{abstract}

Keywords: dog, pharmacologic ablation, gentamicin, glaucoma

\section{INTRODUÇÃO}

O glaucoma canino é uma afecção oftalmológica importante, destacando-se principalmente por sua incidência relativamente elevada em certas raças de cães, nas quais promove elevação da pressão intra-ocular (PIO) suficiente para lesar o nervo óptico e induzir a cegueira temporária ou permanente. É considerado uma emergência ocular, na qual o principal objetivo da terapia é a

Recebido em 30 de dezembro de 2005

Aceito em 26 de fevereiro de 2007

E-mail: j_leandro2000@yahoo.com.br 
redução da PIO, a fim de minimizar a lesão ao nervo óptico (Martin, 1995; Gelatt e Brooks, 1999; Wouk et al., 1999).

Embora o controle medicamentoso seja possível durante um período de tempo limitado, a maioria dos casos de glaucoma em cães requer a associação deste com conduta cirúrgica para o controle em longo prazo da PIO (Cook, 1997). Os procedimentos cirúrgicos antiglaucomatosos em cães são divididos em técnicas que reduzem a produção de humor aquoso pela destruição parcial do corpo ciliar e procedimentos que aumentam seu escoamento por meio de vias alternativas de drenagem (Gelatt e Brooks, 1999).

Devido à freqüente apresentação tardia dos cães na clínica de pequenos animais associada a terapias médicas e cirúrgicas limitadas para o glaucoma na medicina veterinária, pacientes com glaucoma incontrolado ou crônico apresentam, geralmente, olhos irreversivelmente cegos, aumentados de tamanho e predispostos a ceratites de exposição, que são condições agudamente dolorosas (Moller et al., 1986; Martin, 1995). Nesses casos, o objetivo da terapia é aliviar a dor ocular, provendo o olho com pouco ou nenhum requerimento de medicação e com uma condição cosmética aceitável (Moller et al., 1986; Gelatt e Brooks, 1999). Dentre os procedimentos passíveis de proporcionar tais objetivos, estão enucleação, inserção de prótese ocular e ablação química do corpo ciliar (Moller et al., 1986; Cook, 1997; Gelatt e Brooks, 1999), este último é um procedimento de salvamento do olho glaucomatoso, que previne a dor ocular, reduzindo seu tamanho até próximo ao normal e provendo uma condição cosmética aceitável ao proprietário.

Apesar de a ablação intravítrea ser uma técnica já descrita (Vainisi et al., 1983; Moller et al., 1986; Clerc e Robberechts, 1992; Bingaman et al., 1994; Bingaman e Krohne, 1994; Peiffer, 1994), a realização do presente trabalho foi estimulada pela possibilidade de avaliarem-se clinicamente os resultados da ablação intravítrea em longo prazo.

Os objetivos foram avaliar clinicamente as alterações oftalmológicas do bulbo ocular, assim como os aspectos relacionados ao comportamento dos animais referentes à dor, em conseqüência do procedimento.

\section{MATERIAL E MÉTODOS}

Foram utilizados 13 cães, de raças e idades variadas, machos e fêmeas, portadores de glaucoma crônico unilateral. $\mathrm{O}$ grupo experimental foi composto por cães atendidos em serviço de oftalmologia em hospital veterinário.

Os cães foram submetidos ao exame oftálmico, em ambiente de luminosidade controlada, dos anexos oculares e das estruturas do segmento anterior do bulbo do olho, por meio de biomicroscopia em lâmpada de fenda ${ }^{1}$. Avaliações complementares também foram realizadas como o teste de Schirmer ${ }^{2}$, da fluoresceína sódica ${ }^{3}$ e tonometria de aplanação ${ }^{4}$. Animais que apresentaram alterações oculares ou sistêmicas que inviabilizassem a realização do procedimento de ablação não foram incluídos na pesquisa.

Foram selecionados cães que apresentavam glaucoma crônico em estágio avançado com olhos irreversivelmente cegos, de acordo com os critérios a seguir: análise do histórico do animal foram incluídos somente animais com histórico prolongado de alterações oculares compatíveis com a afecção em estudo - cujo período foi superior a três semanas; acuidade visual do olho acometido, após a oclusão do olho contralateral não glaucomatoso, com inclusão apenas de cães com déficit visual do olho acometido; animais que apresentaram os sinais clínicos compatíveis com a presença de glaucoma crônico com acometimento unilateral, observando-se presença de buftalmia ou hidroftalmia e valores da tonometria de aplanação superiores a $33 \mathrm{mmHg}$.

$\mathrm{Na}$ presença de meios oculares suficientemente transparentes, foram avaliadas, mediante oftalmoscopia direta, alterações compatíveis com glaucoma crônico no nervo óptico e retina, tais como disco óptico escavado e cinza a esbranquiçado e hiperreflexia tapetal com atenuação dos vasos retinianos.

\footnotetext{
${ }^{1}$ modelo SL-450, Nidek Co., Japan

${ }^{2}$ Teste de Schirmer - Ophthalmos - Brasil

${ }^{3}$ Fluoresceína - Allergan - Brasil

${ }^{4}$ modelo Tonopen XL, Mentor, Netherlands
} 
Outros sinais clínicos relacionados foram observados, não sendo a sua ausência critérios eliminatórios, como presença de rupturas de Descemet (estrias de Haab`s); graus variáveis de edema de córnea; congestão de vasos episclerais; midríase fixa; sinéquias anterior ou posterior; luxação ou subluxação da lente e catarata.

Em ambos os olhos de todos os animais selecionados, foi realizado exame físico e oftálmico, imediatamente antes da ablação uveal intravítrea (momento zero $-\mathrm{M}_{0}$ ) e após uma semana (momento $1-\mathrm{M}_{1}$ ), quatro $\left(\mathrm{M}_{4}\right)$ e oito semanas $\left(\mathrm{M}_{8}\right)$. $\mathrm{O}$ tempo de seguimento estendeuse até 12 semanas $\left(\mathrm{M}_{12}\right)$ em 12 animais, 24 semanas $\left(\mathrm{M}_{24}\right)$ em 10 animais e 48 semanas $\left(\mathrm{M}_{48}\right)$ em cinco animais.

Após as avaliações iniciais $\left(\mathrm{M}_{0}\right)$, os cães foram pré-anestesiados com cloridrato de levomepromazina $^{5}$, na dose de $0,5 \mathrm{mg} / \mathrm{kg}$, associado ao butorfanol $^{6}, \quad 0,2 \mathrm{mg} / \mathrm{kg}$, via intramuscular. A seguir, foi feita anestesia geral intravenosa de curta duração com propofol ${ }^{7}$, na dose de $5 \mathrm{mg} / \mathrm{kg}$. No olho acometido por glaucoma crônico, procedeu-se à anti-sepsia da superfície ocular e do saco conjuntival com solução de polivinilpirrolidona a 1:50 e emprego de pano de campo oftálmico estéril. Foi introduzida agulha hipodérmica $30 \times 7$ acoplada a uma seringa de $1 \mathrm{ml}$, a seis milímetros posteriormente ao limbo, posicionada entre 9 e 10 horas, sendo feita aspiração de cerca de $0,8 \mathrm{ml}$ de humor vítreo. A seringa foi trocada, sendo injetado $0,8 \mathrm{ml}$ de uma solução contendo $0,5 \mathrm{ml}$ de sulfato de gentamicina ${ }^{8}(20 \mathrm{mg})$ associada a $0,3 \mathrm{ml}$ de dexametasona ${ }^{9}(1,2 \mathrm{mg})$.

Uma injeção subconjuntival de $0,3 \mathrm{ml}$ de gentamicina $(12 \mathrm{mg})$ e $0,3 \mathrm{ml}$ de dexametasona $(1,2 \mathrm{mg})$ foi aplicada após o procedimento de ablação. Todos os animais foram mantidos com colar do tipo elizabetano, sendo prescrito colírio de acetato de prednisolona $1 \%{ }^{10}$, seis vezes ao dia, durante 30 dias, e $2,2 \mathrm{mg} / \mathrm{kg}$ de carprofeno ${ }^{11}$, via oral, durante cinco dias.

\footnotetext{
${ }^{5}$ Neozine - Rhodia Pharma - Brasil

${ }^{6}$ Torbugesic - Fort Dodge - Brasil

${ }^{7}$ Diprivan - Zeneca - Brasil

${ }^{8}$ Garamicina - Ariston - Brasil

${ }^{9}$ Decadron - Prodome - Brasil

${ }^{10}$ Pred Fort - Allergan - Brasil

${ }^{11}$ Rimadyl - Pfizer - Brasil
}

Os animais foram avaliados por meio de exame oftálmico nos diferentes momentos, mediante utilização de foco de luz e biomicroscopia em lâmpada de fenda, para observação da evolução clínica dos sinais de secreção ocular, blefaroespasmo, quemose, hiperemia conjuntival, hifema e alterações corneanas, como opacidade, pigmentação, neovascularização e pannus. Essas alterações foram quali-quantificadas em: 0 - ausente; 1 - discreto; 2 - moderado; 3 - intenso (Munger, 2002). Além disso, foram avaliados aspectos relacionados ao comportamento dos animais, principalmente aqueles referentes à dor, tais como diminuição ou aumento do apetite, quali-quantificados em 0 - normal; 1 - diminuído; 2 - aumentado; e variações do peso relativo corporal, definido por:

$$
\begin{gathered}
\text { Peso relativo }=\frac{\mathrm{P}_{\mathrm{i}}-\mathrm{P}_{0}}{\mathrm{P}_{0}} \text {, em que: } \\
\mathrm{Pi}=\text { peso em cada momento }(\mathrm{kg}) \\
\mathrm{P}_{0}=\text { peso inicial }(\mathrm{kg})
\end{gathered}
$$

Usou-se o teste não paramétrico de Friedman, visando à comparação do efeito do tratamento nos diferentes momentos, com nível de significância de $5 \%$ de probabilidade (Zar, 1996).

\section{RESULTADOS E DISCUSSÃO}

$\mathrm{Na}$ Tab. 1 encontram-se as estatísticas das diferentes variáveis analisadas, representadas pelas medianas.

Não se verificou diferença significativa entre momentos em relação aos escores de secreção ocular no olho acometido. Entretanto, verificouse clinicamente uma intensidade discreta na primeira semana após o procedimento de ablação, que evoluiu para diminuição a partir de oito semanas pós-ablação; acredita-se que ela foi causada pelo desconforto referente à lesão tecidual da realização do procedimento, diminuindo após o controle da inflamação ocular. 


\section{Chiurciu et al.}

Tabela 1. Medianas dos escores das variáveis estudadas, em cães submetidos à ablação intravítrea com glutamicina, segundo os momentos

\begin{tabular}{lccccccc}
\hline \multicolumn{1}{c}{ Variável } & \multicolumn{7}{c}{ Momentos $(\mathrm{M})$ semanas } \\
\hline Secreção ocular & $\mathrm{M}_{0}$ & $\mathrm{M}_{1}$ & $\mathrm{M}_{4}$ & $\mathrm{M}_{8}$ & $\mathrm{M}_{12}$ & $\mathrm{M}_{24}$ & $\mathrm{M}_{48}$ \\
Hiperemia conjuntival & $0,0 \mathrm{a}$ & $1,0 \mathrm{a}$ & $0,0 \mathrm{a}$ & $0,0 \mathrm{a}$ & $0,0 \mathrm{a}$ & $0,0 \mathrm{a}$ & $0,0 \mathrm{a}$ \\
Quemose & $2,0 \mathrm{a}$ & $2,0 \mathrm{ab}$ & $1,5 \mathrm{ab}$ & $1,0 \mathrm{ab}$ & $1,0 \mathrm{~b}$ & $1,0 \mathrm{~b}$ & $1,0 \mathrm{~b}$ \\
Blefaroespasmo & $0,0 \mathrm{a}$ & $0,0 \mathrm{a}$ & $0,0 \mathrm{a}$ & $0,0 \mathrm{a}$ & $0,0 \mathrm{a}$ & $0,0 \mathrm{a}$ & $0,0 \mathrm{a}$ \\
Hifema & $0,0 \mathrm{a}$ & $0,0 \mathrm{a}$ & $0,0 \mathrm{a}$ & $0,0 \mathrm{a}$ & $0,0 \mathrm{a}$ & $0,0 \mathrm{a}$ & $0,0 \mathrm{a}$ \\
Opacidade corneana & $0,0 \mathrm{a}$ & $0,0 \mathrm{a}$ & $0,0 \mathrm{a}$ & $0,0 \mathrm{a}$ & $0,0 \mathrm{a}$ & $0,0 \mathrm{a}$ & $0,0 \mathrm{a}$ \\
Neovascularização & $1,0 \mathrm{ab}$ & $1,0 \mathrm{a}$ & $1,0 \mathrm{ab}$ & $1,0 \mathrm{ab}$ & $1,5 \mathrm{~b}$ & $2,0 \mathrm{~b}$ & $2,0 \mathrm{ab}$ \\
Pigmentação corneana & $0,0 \mathrm{a}$ & $0,0 \mathrm{a}$ & $0,0 \mathrm{a}$ & $0,0 \mathrm{a}$ & $0,5 \mathrm{a}$ & $0,5 \mathrm{a}$ & $0,0 \mathrm{a}$ \\
Pannus corneano & $0,0 \mathrm{a}$ & $0,0 \mathrm{a}$ & $0,0 \mathrm{a}$ & $0,0 \mathrm{a}$ & $1,0 \mathrm{a}$ & $1,0 \mathrm{a}$ & $1,0 \mathrm{a}$ \\
\hline
\end{tabular}

Letras distintas na linha indicam diferenças entre momentos $(\mathrm{P}<0,05)$

Quanto aos escores de hiperemia conjuntival, houve diferença significativa do $\mathrm{M}_{0}$ em relação aos $\mathrm{M}_{12}, \mathrm{M}_{24}$ e $\mathrm{M}_{48}$, portanto, $\mathrm{M}_{0}$ apresentou maior grau de hiperemia conjuntival que os três últimos. Houve evolução regressiva de moderada, na fase inicial $\left(\mathrm{M}_{0}\right)$, para discreta ao longo do experimento, sendo significativa a partir de 12 semanas pós-ablação. Acredita-se que essa melhora seja decorrente do procedimento de ablação associado à diminuição da inflamação ocular, proporcionada pela instilação tópica de corticosteróide, semelhante ao descrito por Moller et al. (1986), que controlaram a inflamação em 15 dos 16 olhos tratados, após um mês de ablação, com o uso de corticosteróide tópico.

Não houve diferença significativa entre momentos, em relação aos escores de hifema, no olho acometido, contudo hifema foi observado em quatro animais, três deles após quatro semanas do procedimento de ablação intravítrea. Segundo Moller et al. (1986), a causa de sangramento, tanto no segmento anterior quanto posterior, deve-se principalmente a trauma mecânico no corpo ciliar causado pela inserção da agulha. Os autores não descartaram a possibilidade de resposta tóxica aguda do tecido uveal frente à gentamicina. As presentes observações também sugerem que essa resposta tóxica deve influenciar no desencadeamento da hemorragia, pois a maioria dos casos de hifema não ocorreu no pós-operatório imediato.

Quanto à opacidade corneana, houve diferença significativa do $M_{1}$ em relação aos $M_{12}$ e $M_{24}$. Nesses momentos, verificou-se opacidade mais acentuada, que estava presente na maioria dos animais antes do procedimento de ablação intravítrea, decorrente da alteração na estrutura da córnea secundária ao aumento da PIO (Mishima, 1982; Gelatt e Brooks, 1999). Houve sensível melhora desse sinal após uma semana de tratamento, decorrente da diminuição significativa da PIO observada nos olhos acometidos. Redução semelhante da opacidade corneana também foi observada por Moller et al. (1986), em dois casos descritos.

Nos momentos mais tardios do experimento, notou-se aumento significativo da opacidade, com progressão de discreta para moderada. Esse fenômeno deveu-se, provavelmente, à diminuição da quantidade de humor aquoso, causando morte das células endoteliais e conseqüente opacificação da córnea (Gelatt e Brooks, 1999). Deve-se levar em consideração uma possível opacificação devido ao efeito tóxico da gentamicina sobre o endotélio, embora a literatura enfatize a toxicidade no tecido retiniano (Mcdonald et al., 1986; Waltz e Margo, 1991; Daily et al., 1995; Loewenstein et al., 2001).

Moller et al. (1986) verificaram relação direta entre diminuição de tamanho ocular e opacificação da córnea, fato observado em três animais do presente estudo, nos quais três olhos apresentaram tamanhos reduzidos e escore severo de opacidade corneana. Outras alterações corneanas, como neovascularização e pigmentação, seguiram o mesmo padrão de evolução da opacidade de córnea, com leve aumento de seus escores após 12 semanas, apesar de não serem estatisticamente diferentes. 
Não se observou diferença significativa entre momentos em relação aos escores, quanto à quemose, ao blefaroespasmo e ao pannus corneano no olho acometido.

Nas variáveis relacionadas à avaliação clínica da dor, como apetite e peso (Tab. 2), a ausência de alterações significativas durante os momentos foi indicativa de que o procedimento de ablação intravítrea, frente aos cuidados pós-aplicação realizados, não promoveu aumento da sensibilidade dolorosa, pois não houve redução de apetite e peso por parte dos animais, embora tenha sido impossível avaliar se a supressão da dor foi devido ao fato de a ablação não se tratar de um procedimento doloroso ou devido à administração conjunta de antiinflamatórios tópico e sistêmico. Verificou-se aumento relativo de peso de cerca de 7,8 a 19,2\% nos momentos 1 a 8 , indicando, biologicamente, maior conforto dos animais. Moller et al. (1986) relataram que 15 entre 16 animais tratados apresentaram-se confortáveis após um mês de ablação, embora o único parâmetro utilizado pelos autores tenha sido a resolução da inflamação ocular.

Tabela 2. Medianas dos escores de apetite e dos valores de peso relativo, em cães submetidos à ablação intravítrea com gentamicina, segundo os momentos

\begin{tabular}{lccccccc}
\hline \multicolumn{1}{c}{ Momentos (M) semanas } \\
\hline Variáveis & $\mathrm{M}_{0}$ & $\mathrm{M}_{1}$ & $\mathrm{M}_{4}$ & $\mathrm{M}_{8}$ & $\mathrm{M}_{12}$ & $\mathrm{M}_{24}$ & $\mathrm{M}_{48}$ \\
\hline Apetite & $0,0 \mathrm{a}$ & $0,0 \mathrm{a}$ & $0,0 \mathrm{a}$ & $0,0 \mathrm{a}$ & $0,0 \mathrm{a}$ & $0,0 \mathrm{a}$ & $0,0 \mathrm{a}$ \\
\hline Peso relativo & $0,008 \mathrm{a}$ & $0,016 \mathrm{a}$ & $0,019 \mathrm{a}$ & $-0,002 \mathrm{a}$ & $-0,028 \mathrm{a}$ & $0,046 \mathrm{a}$ & $0,008 \mathrm{a}$ \\
\hline
\end{tabular}

Não houve diferenças entre os momentos $(\mathrm{P}>0,05)$

\section{CONCLUSÕES}

A ablação uveal intravítrea promoveu diminuição da hiperemia conjuntival causada pelo glaucoma crônico, além da resolução clínica da inflamação ocular, porém teve como conseqüência um aumento de opacidade corneana.

\section{REFERÊNCIAS BIBLIOGRÁFICAS}

BINGAMAN, D.P.; KROHNE, S.G. Intraocular gentamicin in glaucoma. Vet. Comp. Ophthalmol., v.4, p.166, 1994

BINGAMAM, D.P.; LINDLEY, D.M.; GLICKMAN, N.W. et al. Intraocular gentamicin and glaucoma: a retrospective study of $60 \mathrm{dog}$ and cat eyes (1985-1993). Vet. Comp. Ophthalmol., v.4, p.113-119, 1994.

CLERC, B.; ROBBERECHTS, C. Treatment of absolute glaucoma by intravitreous injection of gentamicin. Clin. Res. Rep. Recueil Med. Vet., v.168, p.97-103, 1992.

COOK, C.S. Surgery of glaucoma. Vet. Clin. N. Am.: Small Anim. Pract., v.27, p.1109-1129, 1997.

DAILY, M.J.; KACHMARYK, M.M.; FOODY, R.J. Successful prevention of visual loss with emergency management following inadvertent intracameral injection of gentamicin. Arch. Ophthalmol., v.113, p.855-856, 1995.

GELATT, K.N.; BROOKS, D.E. The canine glaucoma. In. GELATT, K.N. Veterinary Ophthalmology. 3.ed. Philadelphia: Lippincott Williams \& Wilkins, 1999. p. 701754.

LOEWENSTEIN, A.; ZEMEL, E.; VERED, Y. et al. Retinal toxicity of gentamicin after subconjunctival injection performed adjacent to thinned sclera. Ophthalmology, v.108, p.759-764, 2001.

MARTIN, C. L. Glaucoma. In: SLATTER, D. Manual de cirurgia de pequenos animais. 2.ed. São Paulo: Manole, 1995. p.1506-1521.

McDONALD, H.R.; SCHATZ, H.; ALLEN, A.W. et al. Retinal toxicity secundary to intraocular gentamicin injection. Ophthalmology, v.93, p.871-877, 1986.

MISHIMA, S. Clinical investigation on the corneal endothelium. Am. J. Ophthalmol., v.93, p.1-29, 1982.

MOLLER, I.; COOK, C. S.; PEIFFER, R. L. et al. Indications for and complications of pharmacological ablation of the ciliary body for the treatment of chronic glaucoma in the dog. J. Am. Anim. Hosp. Assoc., v.22, p.319326, 1986.

MUNGER, R.J. Veterinary ophthalmology in laboratory animal studies. Vet. Ophthalmol., v.5, p.167-175, 2002.

PEIFFER, R.L. Intraocular gentamicin in glaucoma. Vet. Comp. Ophthalmol., v.4, p.166, 1994.

VAINISI, S.J.; SCHMIDT, G.M.; WEST, C.S. et al. Intraocular gentamicin for the control of endophthalmitis and glaucoma in animals. Trans. Am. Coll. Vet. Ophthalmol., v.14, n.134, 1983.

WALTZ, K.; MARGO, C.E. Intraocular gentamicin toxicity. Arch. Ophthalmol., v.109, p.911, 1991.

WOUK, A.F.P.F.; CÍRIO, S.; KASECKER, G.G. et al. Novo modelo experimental de glaucoma em cão para o estudo da cicatrização após cirurgia filtrante associada ao uso de agente antifibrótico. Arch. Vet. Sci., v.4, p.103-109, 1999.

ZAR, J.H. (Ed). Biostatistical analysis. New Jersey: PrenticeHall, 1996. 718p. 